\title{
The Value of Area Under the Curve Analysis as an Outcome Measure in the Assessment of a Continuous Microdosing Fluocinolone Acetonide Implant (ILUVIEN ${ }^{\circledR}$ )
}

\author{
Sepehr Bahadorani ${ }^{1}$ and Michael A Singer ${ }^{2}$ \\ 1. University of Texas Health Science Center at San Antonio, San Antonio, Texas, US; 2. Medical Center Ophthalmology Associates, San Antonio, Texas, uS
}

DOl: https://doi.org/10.17925/USOR.2017.10.01.46

\begin{abstract}
$\mathrm{T}$ he treatment of diabetic macular edema (DME) has become one of the most challenging management issues faced by ophthalmologists in daily practice. This is partly due to the multifactorial nature of DME, progression of the disease and the clinical capacity needed to administer intravitreal injections. In recent years, the treatment options for DME have expanded to include intravitreal injections of anti-vascular endothelial growth factor (VEGF) agents and steroids, and most recently, the use of intravitreal implants. In determining clinically relevant endpoints for clinical studies of these treatments, it is important to consider the patient's journey and experience with a treatment over a specific time-period rather than isolated outcomes at a pre-specified time-point. Area under the curve (AUC) is an ideal tool for data analysis particularly when it comes to sustained release therapies such as dexamethasone (Ozurdex ${ }^{\circledR}$, Allergan Inc., California, US) and intravitreal fluocinolone acetonide implant $\left(0.2 \mu \mathrm{g} /\right.$ day FAc implant; Iluvien ${ }^{\circledast}$, Alimera Sciences Inc., Georgia, US). Unlike single time-point outcomes, AUC analysis provides the average letters gained per day over the entire treatment period, providing a better measure of long-term effectiveness. A recent analysis using the AUC approach highlights the significant visual acuity benefit resulting from the $0.2 \mu \mathrm{g} /$ day fluocinolone acetonide (FAC) implant during the FAME (Fluocinolone Acetonide for Macular Edema) trials. A step-by-step instruction is included in this article that allows statistical analysis of the AUC data from both functional and anatomical outcomes, using a free software tool to further facilitate the use of this technique for future investigators.
\end{abstract}

\section{Keywords}

Diabetic macular edema, intravitreal implants, ILUVIEN

Disclosure: Sepehr Bahadorani has nothing to declare in relation to this article. Michael Singer would like to disclose consultant speaker research for Allergan, consultant speaker research for Genentech, speaker research for Regeneron and consultant research for Alimera Sciences.

Acknowledgements: The authors would like to acknowledge the help from Aron Trevino, who confirmed the accuracy of the AUC R code. Medical writing support was provided by Katrina Mountfort and James Gilbart at Touch Medical Media, supported by Alimera Sciences.

Authorship: All named authors meet the International Committee of Medical Journal Editors (ICMJE) criteria for authorship of this manuscript, take responsibility for the integrity of the work as a whole, and have given final approval to the version to be published.

Open Access: This article is published under the Creative commons Attribution Noncommercial License, which permits any noncommercial use, distribution, adaptation, and reproduction provided the original author(s) and source are given appropriate credit.

Received: March 7, 2017

Accepted: March 22, 2017

Citation: US Ophthalmic Review, 2017;10(1):46-51

Corresponding Author: Michael A Singer, 9157 Huebner Road, San Antonio, TX 78240, US. E: msinger11@me.com

Support: The publication of this article was supported by Alimera Sciences, who was given the opportunity to review the article for scientific accuracy before submission. Any resulting changes were made at the authors' discretion.
Vascular exposure to hyperglycemia over extended periods, destroys the retinal endothelial cell tight junctions and leads to the development of macular edema with consequent visual loss. ${ }^{1}$ Indeed, diabetic macular edema (DME) is the most common cause of visual impairment in diabetic patients. ${ }^{2}$ At the same time, considering that the incidence of diabetes is on the rise, it becomes prudent to effectively address DME. According to the World Health Organization (WHO) study, the global incidence of diabetes has risen from 108 million in 1980 to 422 million in 2014 and attributes to $2.6 \%$ of global blindness. ${ }^{3}$ These estimates are expected to rise further due to the increasing prevalence of diabetes, ageing of the population and the increasing life expectancy of those with diabetes.

Until recently, the standard of care for DME was laser photocoagulation, which slows progression of vision loss but rarely results in improvement of vision, even after 3 years of treatment. According to the Early Treatment of Diabetic Retinopathy Study (ETDRS), laser treatment of clinically significant macular edema (CSME) decreases the risk of moderate vision impairment (15 letters) by half over 3 years. ${ }^{4}$ Nonetheless, the introduction of intravitreal pharmacologic agents, namely the anti-vascular endothelial growth factor (VEGF) agents and corticosteroids, has changed the prognosis of DME from stabilization of vision to improvement.

\section{Pathogenesis and treatment}

The pathogenesis of DME is attributed to the destruction of endothelial cell tight junctions but more specifically, it is thought that, as a consequence of non-perfusion and hypoxia in diabetic retinopathy, production of VEGF and other inflammatory cytokines attributes to the distortion of vascular barriers. 
The endothelial cells and their tight junctions act as a barrier to maintain the water and protein balance between the intravascular and extravascular compartments. Nevertheless, VEGF and other inflammatory cytokines disrupt this barrier function by altering the structure of the cytoskeleton and/or by affecting the expression of adhesive proteins at endothelial junctions. ${ }^{5-7}$ Therefore, not surprisingly, DME treatments target the direct intravitreal injection of steroids and anti-VEGF agents. Current intravitreal treatments include short acting options such as intravitreal triamcinolone, ${ }^{8}$ Ozurdex(R) (Allergan Inc., Irvine, CA, US), a dexamethasone implant, ${ }^{9}$ antiVEGF agents including ranibizumab, ${ }^{12-14}$ bevacizumab, ${ }^{15}$ pegaptanib, ${ }^{16}$ and aflibercept, ${ }^{17}$ and longer-acting options such as Iluvien (Alimera Sciences Inc., Alpharetta, GA, US) which releases $0.2 \mu \mathrm{g}$ /day fluocinolone acetonide (FAC) for 36 months. ${ }^{10,11}$

\section{Bevacizumab}

Bevacizumab (Avastin ${ }^{\circledR}$, Genentech, San Francisco, CA, US) is a full-length humanized monoclonal antibody that binds to all the isoforms of VEGF.18,19 According to the BOLT (Bevacizumab Or Laser Therapy) trial, in patients with CSME and treated with bevacizumab, mean best corrected visual acuity (BCVA) was significantly higher (64.4 \pm 13.3 ; mean \pm standard deviation [SD] ETDRS letters) than patients treated with laser (54.8 \pm 12.6 ETDRS letters). This study showed that the bevacizumab arm gained a median of 9 ETDRS letters in comparison to 2.5 letters in laser treatment group $(p=0.005) .{ }^{15}$ Additionally, the Diabetic Retinopathy Clinical Research (DRCR) study reveals a more robust reduction of central subfield thickness (CST) in the bevacizumab arm compared to the laser arm..$^{20}$

\section{Ranibizumab}

Ranibizumab (Lucentis ${ }^{\circledR}$, Genentech, San Francisco, CA, US) is a humanized, affinity-matured, monoclonal antibody Fab fragment that binds to all isoforms of human VEGF-A. ${ }^{18}$ Unlike the full-length antibodies (e.g., bevacizumab), ranibizumab's lack of the $\mathrm{Fc}$ region makes the medication more permeable to retinal pigment epithelium (RPE) layers and thus, theoretically, ranibizumab is more effective than bevacizumab in reaching the choroidal neovascularization beneath the RPE layer. ${ }^{21}$ Nevertheless, considering these structural differences, the half-life of ranibizumab is markedly shorter than bevacizumab and the medication clears more quickly from systemic circulation following an intravitreal injection with half-lives of 2 hours and 20 days, respectively. ${ }^{22-25}$

The READ-2 study (Ranibizumab for Edema of the mAcula in Diabetes) was a pioneering work that demonstrated a significantly better BCVA outcome for DME patients following ranibizumab (0.5 mg) injection compared to laser treatment: A BCVA gain of 7.24 letters in the ranibizumab arm compared to a loss of 0.43 letters in the laser arm at month $6 .{ }^{26}$ Later studies continued to support the effectiveness of ranibizumab in treatment of DME as well as its superiority over laser treatment. According to the RESTORE study, in comparison to laser treatment alone, ranibizumab (0.5 $\mathrm{mg}$ ) monotherapy over a 12 months period results in a significantly greater proportion of DME patients with a BCVA letter score $\geq 15 .{ }^{14}$ The RESOLVE study also shows that, in comparison to sham and after a 12-month period, three monthly intravitreal injections of ranibizumab $(0.3$ or $0.5 \mathrm{mg}$ ) significantly improved the BCVA from baseline by 10.3 \pm 9.1 letters. Also, in the ranibizumab group, there was a significant reduction in central retinal thickness $(194.2 \pm 135.1 \mu \mathrm{m})$ compared to the sham group $(48.4 \pm 153.4 \mu \mathrm{m}) .^{13}$ RIDE and RISE are identical phase III studies that compared the efficacy and safety of sham and ranibizumab injections in patients with DME. In
RIDE the proportion of patients gaining $\geq 15$ ETDRS letters in BCVA from baseline at month 24, were as follows: $19.2 \%$ (sham or cross over to 0.5 $\mathrm{mg}), 36.8 \%(0.3 \mathrm{mg})$, and $40.2 \%(0.5 \mathrm{mg})$. On the same note, the RIDE study shows a $22.0 \%$ (sham/0.5 mg), 51.2\% (0.3 mg), and $41.6 \%$ (0.5 mg) improvements in BCVA. ${ }^{12}$

\section{Pegaptanib}

Pegaptanib (Macugen ${ }^{\circledR}$, Gilead Sciences Inc., San Dimas, CA, US) is another anti-angiogenic antibody that specifically binds and blocks the activity of extracellular VEGF-A165 isoform, which is the most potent and abundant form of VEGF. ${ }^{27}$ This treatment is administered as monthly or bimonthly intravitreous injections. The combined analysis of multiple trials demonstrated a lower loss of visual acuity at 12 months in patients treated with pegaptanib (0.3 mg: 70\% of patients; $1.0 \mathrm{mg}$ : $71 \%$ of patients; $3.0 \mathrm{mg}$ : $65 \%$ of patients) treatment compared to the sham injection group $(55 \%) .{ }^{28}$

\section{Aflibercept}

Aflibercept (Eylea ${ }^{\circledR}$, Regeneron Pharmaceutical, Tarrytown, NY, US) is a recombinant fusion protein that contains portions of human VEGF receptor fused to the FC domain of human immunoglobulin G (IgG)1 antibody. ${ }^{29}$ The DA VINCI study demonstrates that aflibercept injection is more effective than the laser at improving BCVA as well as CST at week 52: aflibercept arm with BCVA gain of 9.7 to 12 letters and CST reduction of 165.4 to $227.4 \mu \mathrm{m}$ compared to laser arm with BCVA loss of 1.3 and CST reduction of $58.4 \mu \mathrm{m} .{ }^{17}$ Likewise, results of two parallel studies, VIVID and VISTA, depict superiority of aflibercept arm a gain in visual acuity compared to the laser arm in patients with DME. letters for ranibizumab.. The mean BCVA gain from baseline to week 148 following treatment with aflibercept or laser was 10.4-10.5 and 1.4 letters in VISTA and 10.3-11.7 and 1.6 letters in VIVID, respectively..$^{30}$ Finally, the DRCR study shows that at 2 years, mean visual acuity improvement are 12.8 letters for aflibercept, 10.0 letters for bevacizumab and 12.3 for ranibizumab. ${ }^{31}$

\section{Triamcinolone}

Inflammation is an important mechanism of DME development and thus, when anti-VEGF agents yield suboptimal results or systemic vascular disorders limit their use, or clinical capacity is limited, steroids become the mainstay of treatment. Intravitreal triamcinolone acetonide has been shown to effectively reduce macular edema in the short term ${ }^{13}$ but is limited by a short duration of action and the need for repeated injections and visits. In addition, intraocular pressure (IOP) rises of $>10 \mathrm{~mm} \mathrm{Hg}(18 \%$ for $1 \mathrm{mg}$; $33 \%$ for $4 \mathrm{mg}$ ) as well as an increased need for cataract surgery (46\% for $1 \mathrm{mg}$; $83 \%$ for $4 \mathrm{mg}$ ) are adverse reactions that discourage most clinicians from using triamcinolone as a preferred agent for first-line treatment of DME. ${ }^{32}$

\section{Dexamethasone implant}

The dexamethasone intravitreal implant $(0.7 \mathrm{mg})$, also known as Dexamethasone Posterior Segment Drug Delivery System (Ozurdex), consists of micronized dexamethasone in a biodegradable copolymer of polylactic-co-glycolic acid which releases dexamethasone into the vitreous over a period up to 6 months as shown in male monkey studies..$^{33}$ The implant is inserted into the vitreous with a 22-gauge needle, ${ }^{34}$ with the goal of reducing the frequency of intravitreal injections. Multiple studies show that the dexamethasone intravitreal implant is an effective means of improving BCVA and reducing macular edema in patients with DME. ${ }^{35-37}$ The adverse reactions of the dexamethasone implant include the need 
Table 1: Best-corrected visual acuity average change from baseline during two studies using a dexamethasone posterior segment drug delivery system (Ozurdex) using an area under the curve approach

\begin{tabular}{|c|c|c|c|c|c|c|c|c|c|}
\hline \multirow{3}{*}{$\begin{array}{l}\text { BCVA change } \\
\text { from baseline }\end{array}$} & \multicolumn{3}{|c|}{ Study 206207-010 } & \multicolumn{3}{|c|}{ Study 206207-011 } & \multicolumn{3}{|c|}{ Pooled studies 206207-010 and -011 } \\
\hline & DEX 700 & DEX 350 & Sham & DEX 700 & DEX 350 & Sham & DEX 700 & DEX 350 & Sham \\
\hline & $n=163$ & $n=166$ & $n=165$ & $\mathrm{n}=188$ & $\mathrm{n}=181$ & $\mathrm{n}=185$ & $n=351$ & $n=347$ & $\mathrm{n}=350$ \\
\hline Mean & $4.1^{*}$ & $4.3^{*}$ & 1.9 & 2.9 & 2.9 & 2.0 & $3.5^{*}$ & $3.6^{*}$ & 2.0 \\
\hline Median & 4.5 & 4.0 & 1.2 & 3.1 & 2.7 & 2.1 & 3.9 & 3.5 & 1.6 \\
\hline
\end{tabular}

* indicates statistically significant ( $p=0.05$ ) difference between DEX 700 or DEX 350 versus sham; BCVA = best-corrected visual acuity; DEX = dexamethasone posterior segment drug delivery system (Ozurdex); SD = standard deviation, either 700 gg or $350 \mu \mathrm{g}$ dose. Note: Average change was calculated using area under the curve (AUC) approach based on observed data, missing values were not imputed. For by-study analyses, $p$-values were based on an analysis of covariance (ANCOVA) with treatment as a factor and baseline value as a covariate. For the pooled analysis, $p$-values were based on an ANCOVA with treatment and study as factors and baseline value as a covariate.

Reproduced with permission from: European medicines Agency EPAR $2014^{47}$

for IOP lowering medications to manage ocular hypertension $(41.5 \%$ for dexamethasone versus $9.1 \%$ for sham) and cataract progression $(67.9 \%$ for dexamethasone versus $20.4 \%$ for sham). ${ }^{38}$

\section{Fluocinolone acetonide implant}

Recently, a new generation of a non-biodegradable, long term intravitreal implant, $0.2 \mu \mathrm{g} /$ day FAc, has become available. The implant is inserted into the vitreous with a 25 -gauge needle. The implant contains $0.19 \mathrm{mg}$ of FAc and releases the drug at a rate of $0.2 \mu \mathrm{g}$ of FAc per day over a period of 36 months. ${ }^{10,39}$ This formulation potentially means fewer injections, visits and improved management of patients. ${ }^{40}$

The next section focuses on the use of area under the curve (AUC) as an effective means of assessing the efficacy of sustained-release medications, most notably the longer-acting steroids, dexamethasone and fluocinolone.

\section{Area under the curve}

AUC is a useful approach for integrating serial assessments of a patient's outcome over the duration of a study. ${ }^{41}$ While area AUC is a fundamental concept in pharmacokinetics, until recently, its use in clinical trials has been largely neglected and only used in a few therapeutic areas. ${ }^{41,42}$

The recent DRCR.net Protocol S study used AUC for assessment of vision outcome in DME treatments for proliferative diabetic retinopathy (PDR). In this trial, the efficacy and safety of intravitreous ranibizumab were compared with panretinal photocoagulation (PRP) in patients with proliferative diabetic retinopathy. ${ }^{43}$ The investigators set the primary outcome as mean visual acuity change at 2 years and the secondary outcome as visual acuity AUC to provide an assessment of vision throughout follow-up. The result of the study show +4.2 difference in AUC visual acuity over two years in the ranibizumab group versus PRP group ( $95 \% \mathrm{Cl},+3.0$ to $+5.4 ; \mathrm{p}<0.001)$. By contrast, the difference in the primary endpoint of mean visual acuity letter improvement at two years was not statistically significant $(+2.2$; $95 \%$ confidence interval $[\mathrm{Cl}],-0.5$ to +5.0 ). These outcomes highlight the importance of AUC analysis in clinical trials, reminding clinicians that AUC can potentially provide a better assessment of the visual acuity gain over the course of a DME trial by indicating the average effect of treatment over time. Likewise, post hoc analyses from the DRCR Network have been performed to assess the change in visual acuity AUC for comparison of aflibercept, bevacizumab, and ranibizumab in the treatment of DME. Results of the study show that eyes with a visual acuity of 20/50 or worse have the greatest improvement over 2 years with aflibercept treatment. ${ }^{44}$
The AUC calculations have also been used in the VISION trial to assess the effectiveness of pegaptanib in the treatment of neovascular age-related macular degeneration. According to this study, the proportions of patients experiencing a loss of $\geq 15$ letters from week 54 to week 102 was half as many in the treatment group as compared with those patients who discontinued pegaptanib. ${ }^{16}$

In particular, considering the gradual decrease in the vitreous concentration of sustained-release medications, the AUC analysis is highly recommended for evaluating the continuous effects of these medications. Indeed, in a pair of 3-year, phase III randomized, shamcontrolled trials, the efficacy of dexamethasone implants $(0.35 \mathrm{mg}$ and $0.7 \mathrm{mg}$ ) in DME patients has been evaluated using the AUC method. ${ }^{45,46}$ The results of these trials have been presented in the European public assessment evaluation of dexamethasone implant (Table 1). ${ }^{47}$ The AUC was the primary efficacy measure. In one of these studies (206207010, $n=494$ ), the average number of BCVA letters gained per day (AUC approach) was significantly greater with dexamethasone implant 700 compared with sham (difference of 2.1,95\% Cl: 0.4-3.8, p=0.016) and with dexamethasone implant 350 compared to sham (difference of 2.3, 95\% Cl: 0.5-4.0, $p=0.010){ }^{47}$ In the other study (206207-011, $n=554$ ) the mean BCVA change from baseline was 2.9 with dexamethasone implant 700 compared to 2.0 with Sham (difference of 0.8 letters, 95\% Cl: -0.9-2.4, $\mathrm{p}=0.366$ ). The AUC was 2.9 with dexamethasone implant 350 compared with 2.0 with sham (difference of $0.7,95 \% \mathrm{Cl}$ : $-0.9-2.4, p=0.396$ ). The differences were not statistically significant indicating that one of the two trials failed its primary endpoint.

These studies demonstrate how the AUC method is regaining popularity, particularly in the context of longer-acting medications. In the MEAD study, the statistically significant difference disappears in terms of BCVA of the total group of dexamethasone implants versus sham group at around month 18 to month 36 months post-initiation of the study (this is presumably due to cataract formation). ${ }^{46}$ Although the BCVA of $0.7 \mathrm{mg}$ implants once again significantly improved from the sham arm at 36-39 months of treatment, this example shows how endpoint mean measurements based on the single time point of final BCVA may be misleading.

The PLACID study is another relevant trial comparing DEX (0.7 mg) combined with laser photocoagulation to laser alone for the treatment of DME. ${ }^{48}$ While there was no statistical difference between the treatment groups at month 12, AUC analysis of BCVA depicts a significantly greater improvement in BCVA of the combined treatment group. 
The most feasible explanation for BCVA decline with the dexamethasone implant is due to a gradual decrease in the vitreous concentration of steroids ${ }^{49}$ and consequent dilution of final outcome measurements towards the end of the trial. Cataract formation could be argued as a source of the decline in terms of vision, but retinal thickness also declines, showing that it is a drug-related effect. Therefore, it is essential to analyze the overall trend of BCVA improvement in studies using sustained-released medications to determine the efficacy of the treatment.

It is worth noting that AUC analysis only interprets the overall statistical significance of a treatment rather than specific time points during the period of study. Hence, this form of analysis may not be suitable for studies that are interested in evaluating early effects rather than long term overall effects, in that early effect of a treatment could be diluted by long term data and lose its statistical significance. Therefore, for these situations, linear or generalized mixed models may be used as an alternative approach to flexible analysis of correlated data.

\section{The $0.2 \mu g /$ day fluocinolone acetonide implant and diabetic macular edema}

considering that the $0.2 \mu \mathrm{g} / \mathrm{day}$ FAc implant is a new generation of sustained-release steroid implant, most of what we know from the effects of this medication comes from European studies, in that, the launch of the $0.2 \mu \mathrm{g} /$ day FAc implant in Europe preceded that in the US by 2 years. Here follows a summary of the $0.2 \mu \mathrm{g} /$ day FAc implant studies.

Safety and efficacy of the $0.2 \mu \mathrm{g} /$ day FAc implant in DME patients has been tested in the FAME trials which included two phase III randomized trials (FAME A and FAME B) comparing 0.5- and 0.2- $\mu \mathrm{g} /$ day implants to sham injection groups. ${ }^{39}$ Combined results of these two studies showed the mean improvement in BCVA letter score from baseline to month 24 was 4.4 and 5.4 for the 0.5- and 0.2- $\mu \mathrm{g}$ /day doses, respectively, compared with 1.7 in the sham group ( $p=0.02$ and $p=0.016$, respectively). At month 36, improvement from baseline in the percentage of patients with a $\geq 15$ ETDRS letter gain continued to be statistically significant for $0.2 \mu \mathrm{g} / \mathrm{day}$ FAc $(28.7 \%)$ and $0.5 \mu \mathrm{g} /$ day FAc $(28.6 \%)$ implants compared with the sham group ( $16.2 \%, p=0.002$ for each). However, phakic patients requiring cataract surgery were more frequent for implant arms (74.9\% for $0.2 \mu \mathrm{g} /$ day FAc and $84.5 \%$, for $0.5 \mu \mathrm{g} /$ day) compared with the sham group (23.1\%, although the cataract formation was higher means that not every patient underwent surgery during the study). Similarly, ocular hypertension-requiring incisional surgery was needed in $3.7 \%, 7.6 \%$, and $0.5 \%$ of the $0.2 \mu \mathrm{g} /$ day, $0.5 \mu \mathrm{g} / \mathrm{day}$, and sham groups, respectively. ${ }^{10}$

Data regarding AUC in the FAME trials was recently presented based on the BCVA letter gain for the $0.2 \mu \mathrm{g}$ /day FAc implant sham (Figure 1). These come from an integrated analysis of the FAME A and FAME $B$ trials showing that at 36 months there was a significant difference in AUC between subjects who received $0.2 \mu \mathrm{g} /$ day FAc implants and sham control injections (5.2 versus 1.6 letters $p<0.001$, Figure 1)..$^{50}$ This benefit was seen in both the FAME A ( 4.5 versus 2.3 letters, $p=0.031$ ) and FAME $B$ (5.8 versus 0.9 letters, $p<0.001)$ trials. For subgroup analysis, subjects with a longer DME duration showed greater BCVA benefit (6.9 versus 1.9, for $0.2 \mu \mathrm{g}$ /day FAc implant versus sham, $\mathrm{p}<0.001)$ compared with those with a shorter duration of DME (2.9 versus $1.4, p=0.03) .{ }^{51}$ In addition, subjects who were pseudophakic at baseline showed greater benefits (7.1 versus $1.7, p<0.001)$ than patients who were phakic at baseline $(4.7$
Figure 1: Summary of the best corrected visual acuity letter score (integrated FAME trials: all subjects)

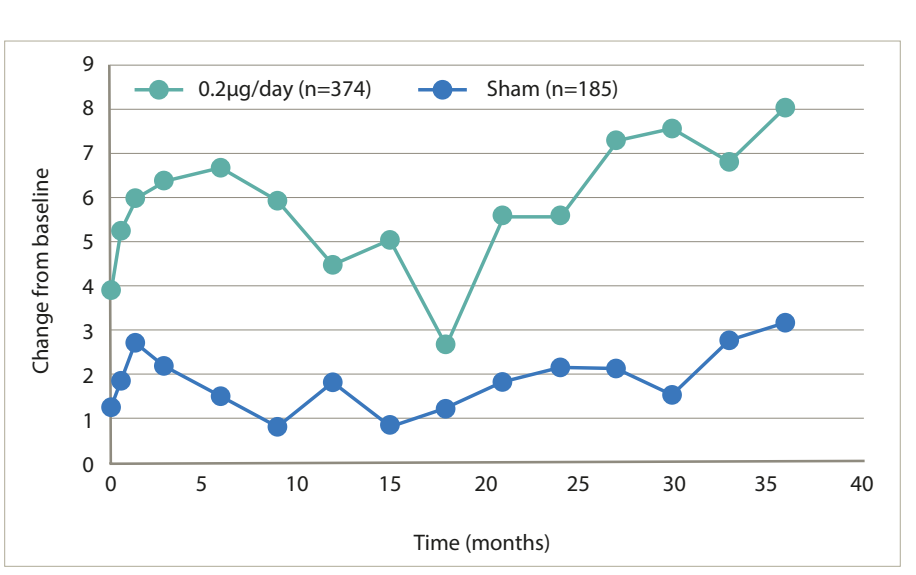

Reproduced with permission from Singer, 2016.50

versus 1.8, $p=0.115) .{ }^{52} \mathrm{An} A U \mathrm{C}$ analysis of the longer DME duration arm in the FAME trials showed numerically similar vision outcomes irrespective of lens status over 36 months (7.8 versus 2.2, $p=0.004$ for those who were pseudophakic at baseline ns 7.4 versus $2.1, p=0.020$ for those who were phakic at baseline and became pseudophakic). ${ }^{53}$

AUC analysis indicates that the $0.2 \mu \mathrm{g} /$ day FAC implant produced significant benefits compared with sham control. The changes in DME were consistently seen in both the FAME A and FAME $B$ trials and were greater $(p=0.029)$ than those observed with other sustained release steroids such as dexamethasone implant 700 but were achieved with fewer injections (1.3 in FAME versus 4.1 in MEAD). This analysis demonstrated that the AUC method captures treatment benefit over the entire dosing period which is particularly appropriate for studies of extended duration.

Besides the FAME trials, another recent study tested the effects of fluocinolone intravitreal implant in DME patients that were resistant to laser and anti-VEGF therapies in a real-life setting. The study tests the effects of the $0.2 \mu \mathrm{g} /$ day FAc implant in two different groups of patients: group 1 with prior laser-only treatment ( $n=7$ eyes), and group 2 comprised of patients with a prior combination of laser and $\geq 3$ monthly anti-VEGF therapy ( $n=10$ eyes). At 12 months following administration of the $0.2 \mu g$ / day FAc implant, DME decreased by $299 \mu \mathrm{m}$ in group 1, and by $251 \mu \mathrm{m}$ in group 2. The analysis used the AUC method to measure changes in BCVA, where $0.2 \mu \mathrm{g} /$ day FAC implant treatment increased the mean area under the curve for pseudophakic patients from baseline to last value by +4.2 letters in group 1 and +9.5 letters in group $2 .{ }^{54}$ This showed a greater improvement in vision in patients who had previously received both laser and anti-VEGF therapy.

\section{Calculation of area under the curve}

The lack of resources or knowledge is one of the main limitations to the use of AUC statistical analyses. Here, with hopes of providing more liberty to future investigators for using this statistical method, we are providing a cost-free approach with step-by-step instructions to analyze the AUC data using the R Statistical Software. The provided codes will analyze the data using one-way ANOVA followed with a pairwise t-student post-hoc test corrected with the Holm's sequential method to reduce the probability of type I error. 
Step 1) Download "AUC.r", "AUC_example.r", and "YWZ.CSV" from the supplementary section of this article.

Step 2) Download R 3.3.2 (or newer versions) from r-cran directory: https://cran.r-project.org/bin/windows/base/

Step 3) Run the installer and follow the instructions.

Step 4) Next, download and install RStudio: https://www.rstudio.com/products/rstudio/download/

Step 5) Execute RStudio.

Step 6) Click the "Open an existing file" icon $\mathbb{B}^{*}$ (or press "Ctrl+O") to open the "AUC.r" as well as "AUC_example.r" script with RStudio.

Step 7) Import the YWZ.csv data by clicking on the "Import Dataset" icon $a^{+}$Import Dataset *. The file must be saved in the R directory (which is usually located in the "documents" folder). Allow the program to install any required packages for importing .csv data. Browse and select your "YWZ" file from R-directory and then click import.

Step 8) Click on the "AUC.r" script थ Auc.rx on top-left corner of the page, highlight the entire text and then click Run $\rightarrow$ Run (if you cannot find the Run icon, then press "Ctrl+R")

Step 9) Click on the "AUC_example.r" script e-auc_example.r $*$, place the cursor on the first line, and execute the codes line-by-line all the way to the bottom by either clicking Run $\rightarrow$ Run or "Ctrl+R".

Step 10) In the console section at left-bottom of the page, $p$-values are listed for statistical comparison of $\mathrm{Y}, \mathrm{W}$, and $\mathrm{Z}$ graphs.

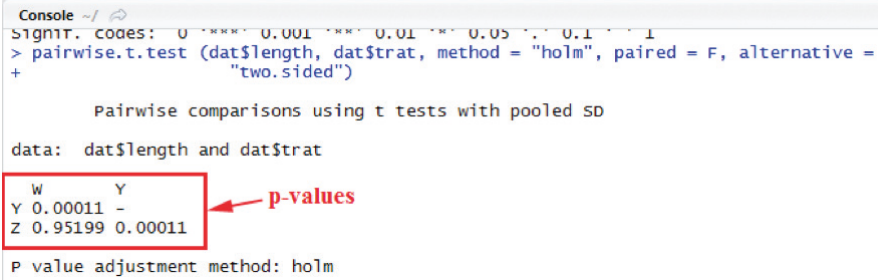

P value adjustment method: holm

Notes for incorporation of your raw data:

- You can modify numbers or add/delete rows in the YWZ.csv file. Please make sure to save the archive and re-import it to $\mathrm{R}$ from R-directory.

- Y, W, and Z each represent a separate variable (e.g., Implants) with their numeric outcome listed in front of them. The numbers in front of "TROT", on the other hand, represent the timeline. All these values can be modified accordingly to incorporate your raw data for AUC calculations.

- If you decide to add or subtract columns in your timeline (TROT), you must also adjust the mm matrix, (i in $2: 12)$, and $c(11,11,11)$ values in the "AUC_example.r" file.

\section{Summary and concluding remarks}

As a result of improved understanding of the pathophysiological processes responsible for endothelial blood-retinal barrier breakdown in DME, new therapeutic options have emerged. Several VEGF inhibitors, including aflibercept, bevacizumab, pegaptanib and ranibizumab, have shown clinical efficacy as intravitreal therapies for DME. In addition, the $0.2 \mu \mathrm{g}$ /day FAc implant and DEX are available as intravitreal implant systems releasing steroid for up to 36 months ${ }^{10,39}$ and 4 to 6 months, ${ }^{55}$ respectively. However, variation in clinical endpoints can hinder comparisons of clinical trials of these agents and often only account for outcomes at a single time-point.

As an alternative approach, the AUC method captures treatment benefit over the entire dosing period. Most DME subjects require treatment for multiple years, thus the AUC method is particularly relevant to assess therapies for DME. Using the AUC method, a significant visual acuity benefit that resulted from $0.2 \mu \mathrm{g} / \mathrm{d}$ FAc versus sham control during the FAME trials was demonstrated. In addition, the similarity between AUC and change in mean BCVA at month 36 supports the consistent benefit with continuous $0.2 \mu \mathrm{g} / \mathrm{d}$ FAc therapy. A recent analysis of data from the FAME trials using the AUC method highlights the sustained benefits of the $0.2 \mu \mathrm{g} /$ day FAC intravitreal implant in the treatment of DME. The AUC method therefore provides a complete assessment of efficacy over time and is particularly relevant for evaluating outcomes over a continued period of therapy. $\square$
1. Antcliff RJ, Marshall J, The pathogenesis of edema in diabetic maculopathy, Semin Ophthalmol, 1999;14:223-32.

2. Bhagat N, Grigorian RA, Tutela A, et al., Diabetic macular edema: pathogenesis and treatment, Surv Ophthalmol, 2009:54:1-32.

3. WHO, Global report on diabetes. Available at: apps.who.int/iris/ bitstream/10665/204871/1/9789241565257_eng.pdf (accessed January 13,2017 )

4. Photocoagulation for diabetic macular edema. Early Treatment Diabetic Retinopathy Study report number 1. Early Treatment Diabetic Retinopathy Study research group, Arch Ophthalmol, 1985;103:1796-806.

5. Rodrigues SF, Granger DN, Blood cells and endothelial barrier function, Tissue Barriers, 2015:3.e978720

6. Campochiaro PA, Peters KG, Targeting Tie2 for Treatment of Diabetic Retinopathy and Diabetic Macular Edema, Curr Diab Rep, 2016;16:126

7. Bolinger MT, Antonetti DA, Moving Past Anti-VEGF: Nove Therapies for Treating Diabetic Retinopathy, Int J MOl SCi 2016;17:pii: E1498

8. Diabetic Retinopathy Clinical Research N, A randomized trial comparing intravitreal triamcinolone acetonide and focal/grid photocoagulation for diabetic macular edema, ophthalmology, 2008:115:1447-9.

9. Haller JA, Kuppermann BD, Blumenkranz MS, et al., Randomized controlled trial of an intravitreous dexamethasone drug delivery system in patients with diabetic macular edema, Arch Ophthalmol, 2010;128:289-96.

10. Campochiaro PA, Brown DM, Pearson A, et al. Sustained delivery fluocinolone acetonide vitreous inserts provide benefit for at least 3 years in patients with diabetic macular edema, Ophthalmology. 2012:119:2125-32.

11. Campochiaro PA, Nguyen QD, Hafiz G, et al., Aqueous levels of fluocinolone acetonide after administration of fluocinolone acetonide inserts or fluocinolone acetonide implants, Ophthalmology, 2013;120:583-7.

12. Brown DM, Nguyen QD, Marcus DM, et al., Long-term Outcomes of Ranibizumab Therapy for Diabetic Macular Edema: The 36-Month Results from Two Phase III Trials: RISE and RIDE Ophthalmology, 2013;120:2013-22.

13. Massin P Bandello F, Garweg JG, et al., Safety and efficacy of ranibizumab in diabetic macular edema (RESOLVE Study): a 12-month, randomized, controlled, double-masked, multicente 12-month, randomized, controlled, double-masked,

14. Mitchell P, Bandello F, Schmidt-Erfurth U, et al., The RESTORE study: ranibizumab monotherapy or combined with laser versus laser monotherapy for diabetic macular edema, Ophthalmology, 2011;118:615-25

15. Rajendram R, Fraser-Bell S, Kaines A, et al., A 2-year prospective randomized controlled trial of intravitreal bevacizumab or laser therapy (BOLT) in the management of diabetic macular edema: therapy (BOLT) in the management of diabetic macular eden

16. Chakravarthy U, Adamis AP, Cunningham ET Jr., et al., Year 2 efficacy results of 2 randomized controlled clinical trials of pegaptanib for neovascular age-related macular degeneration, Ophthalmology, 2006;113:1508 e1-25.

17. Do DV, Nguyen QD, Boyer D, et al., One-year outcomes of the da Vinci Study of VEGF Trap-Eye in eyes with diabetic macular edema, Ophthalmology, 2012:119:1658-65.

18. Lien $\mathrm{S}$, Lowman HB, Therapeutic anti-VEGF antibodies, Handb Exp Pharmacol, 2008:131-50.

19. Ranieri $G$, Patruno $R$, Ruggieri $E$, et al., Vascular endothelial growth factor (VEGF) as a target of bevacizumab in cancer: from the biology to the clinic, Curr Med Chem, 2006;13:1845-57.

20. Scott IU, Edwards AR, Beck RW, et al., A phase II randomized clinical trial of intravitreal bevacizumab for diabetic macular edema, Ophthalmology, 2007:114:1860-7.

21. Terasaki H, Sakamoto T, Shirasawa M, et al., Penetration of bevacizumab and ranibizumab through retinal pigment epithelia layer in vitro, Retina, 2015;35:1007-15.

22. Genetech Inc., Avastin (bevacizumab) solution for intravenous infusion prescribing information., 2016. Available at: www.gen com/download/pdf/avastin_prescribing.pdf (accessed Mar 7 . 2017).
23. Avery RL, Castellarin AA, Steinle NC, et al., Systemic pharmacokinetics following intravitreal injections of ranibizumab, bevacizumab or aflibercept in patients with neovascular AMD, Br J Ophthalmol, 2014:98:1636-41.

24. Ferrara N, Damico L, Shams $\mathrm{N}$, et al., Development of ranibizumab, an anti-vascular endothelial growth factor antigen binding fragment, as therapy for neovascular age-related macular degeneration, Retina, 2006;26:859-70.

25. Xu L, Lu T, Tuomi L, et al., Pharmacokinetics of ranibizumab in patients with neovascular age-related macular degeneration: a population approach, Invest Ophthalmol Vis Sci, 2013;54:1616-24.

26. Nguyen QD, Shah SM, Heier JS, et al., Primary End Point (Six Months) Results of the Ranibizumab for Edema of the mAcula in diabetes (READ-2) study, Ophthalmology, 2009:116:2175-81 e1.

27. Pozarowska D, Pozarowski P, The era of anti-vascular endothelial growth factor (VEGF) drugs in ophthalmology, VEGF and anti-VEGF therapy, Cent Eur J Immunol, 2016;41:311-6.

28. Colquitt JL, Jones J, Tan SC, et al., Ranibizumab and pegaptanib for the treatment of age-related macular degeneration: a systematic review and economic evaluation, Health Technol Assess, 2008;12:iii-iv, ix-201.

29. Ciombor KK, Berlin J, Aflibercept--a decoy VEGF receptor, Curr Oncol Rep, 2014:16:368.

30. Heier JS, Korobelnik JF, Brown DM, et al., Intravitreal Aflibercept fo Diabetic Macular Edema: 148-Week Results from the VISTA and VIVID Studies, Ophthalmology, 2016;123:2376-85.

31. Wells JA, Glassman AR, Ayala AR, et al., Aflibercept, Bevacizumab, or Ranibizumab for Diabetic Macular Edema: Two-Year Results from a Comparative Effectiveness Randomized Clinical Trial Ophthalmology, 2016;123:1351-9.

32. Diabetic Retinopathy Clinical Research N, Beck RW, Edwards AR, et al., Three-year follow-up of a randomized trial comparing focal/ grid photocoagulation and intravitreal triamcinolone for diabetic macular edema, Arch Ophthalmol, 2009;127:245-51.

33. Chang-Lin JE, Attar M, Acheampong AA, et al., Pharmacokinetics and pharmacodynamics of a sustained-release dexamethasone intravitreal implant, Invest Ophthalmol Vis Sci, 2011;52:80-6. 
34. FDA, Ozurdex - Prescribing information NDA 22315/S-009, 2014. Available at: www.accessdata.fda.gov/drugsatfda_docs/ label/2014/022315s009|bl.pdf date (accessed March 22, 2017).

35. Pacella F, Romano MR, Turchetti P, et al., An eighteen-month follow-up study on the effects of Intravitreal Dexamethasone Implant in diabetic macular edema refractory to anti-VEGF therapy, Int J Ophthalmol, 2016;9:1427-32.

36. Yucel OE, Can E, Ozturk HE, et al., Dexamethasone Implant in Chronic Diabetic Macular Edema Resistant to Intravitreal Ranibizumab Treatment, Ophthalmic Res, 2017;57:161-165

37. Aknin I, Melki L, Longitudinal Study of Sustained-Release Dexamethasone Intravitreal Implant in Patients with Diabetic Macular Edema, Ophthalmologica, 2016;235:187-8.

38. Hall J, Correspondence, Retina, 2017:37:e34-e7.

39. Campochiaro PA, Brown DM, Pearson A, et al., Long-term benefit of sustained-delivery fluocinolone acetonide vitreous inserts for diabetic macular edema, Ophthalmology, 2011;118:626-35 e2.

40. Mourtzoukos $\mathrm{S}$, The treatment of diabetic macular oedema (DMO) in UK real-life clinical practice with ILUVIEN® (fluocinolone acetonide) - its impact on current clinical practice, Exp Rev Ophthalmol, 2017:12:95-7.

41. Pham B, Cranney A, Boers M, et al., Validity of area-under-thecurve analysis to summarize effect in rheumatoid arthritis clinical trials, J Rheumatol, 1999;26:712-6.

42. Cappelleri JC, Bushmakin AG, Zlateva G, et al., Pain responder analysis: use of area under the curve to enhance interpretation of clinical trial results, Pain Pract, 2009:9:348-53.
43. Gross JG, Glassman AR, Jampol LM, et al., Panretinal photocoagulation vs intravitreous ranibizumab for proliferative diabetic retinopathy: a randomized clinical trial, JAMA, 2015;314:2137-46.

44. Jampol LM, Glassman AR, Bressler NM, et al., Anti-vascular endothelial growth factor comparative effectiveness trial for diabetic macular edema: additional efficacy post hoc analyses of a randomized clinical trial, JAMA Ophthalmol, 2016;134.

45. Boyer DS, Yoon YH, Belfort R Jr., et al., Three-year, randomized, sham-controlled trial of dexamethasone intravitreal implant in patients with diabetic macular edema, Ophthalmology, 2014;121:1904-14.

46. Augustin AJ, Kuppermann BD, Lanzetta P, et al., Dexamethasone intravitreal implant in previously treated patients with diabetic macular edema: subgroup analysis of the MEAD study, BMC macular edema: subgrou

47. European Medicines Agency, Assessment report (EPAR) Ozurdex - International non-proprietary name: DEXAMETHASONE Procedure No. EMEA/H/C/001140/II/0015 EMA/492068/2014, 2014. Available at: www.ema.europa.eu/docs/en_GB/document library/EPAR_-_Assessment_Report_-_Variation/human/001140/ WC500173294.pdf (accessed March 2, 2017)

48. Callanan DG, Gupta S, Boyer DS, et al., Dexamethasone intravitreal implant in combination with laser photocoagulation for the treatment of diffuse diabetic macular edema, Ophthalmology, 2013;120:1843-51.

49. Herrero-Vanrell R, Cardillo JA, Kuppermann BD, Clinica applications of the sustained-release dexamethasone implant for treatment of macular edema, Clin Ophthalmol, 2011;5:139-46

50. Singer MA, Efficacy Assessment of the $0.2 \mu \mathrm{g} /$ day Fluocinolone Acetonide (FAC) Intravitreal Implants vs. Sham Control Using the Area Under the Curve (AUC) Method (FAME), Presented at the American Society of Retinal Physicians (ASRS) 2016 Annual Meeting, August 10-14, 2016, San Francisco, US.

51. Kuppermann B, Fluocinolone acetonide $0.2 \mu \mathrm{g} /$ day IMPLANT (iluvien) area under the curve (AUC) pharmacodynamics The CONSTANT Analysis: AUC comparisons with Protocol I ranibizumab, deferred laser, Presented at: ISOPT Clinical, Rome, Italy, Dec 1-3, 2016

52. Singer $\mathrm{M}$, Efficacy assessment of the $0.2 \mathrm{mg} /$ day fluocinolone acetonide (FAC) intravitreal implants vs. sham control using the area under the curve (AUC) method, Presented at: ASRS, San area under the curve (AUC) meth
Francisco, US, Aug 9-14, 2016.

53. Elaraoud I, Maya J, Narendran $N$,et al., Efficacy assessment of ILUVIEN (0.2 $\mu \mathrm{g} / \mathrm{d}$ Fluocinolone Acetonide) using the area under the curve method, Presented at EURETINA, Copenhagen, Denmark, Sep 8-11, 2016

54. Massin P, Erginay A, Dupas B, et al., Efficacy and safety of sustained-delivery fluocinolone acetonide intravitreal implant in patients with chronic diabetic macular edema insufficiently responsive to available therapies: a real-life study, Clin Ophthalmol, 2016;10:1257-64.

55. Palla S, Biswas J, Nagesha CK, Efficacy of Ozurdex implant in treatment of noninfectious intermediate uveitis, Indian Ophthalmol, 2015;63:767-70. 